\title{
Surface-Confined Self-Assembly of Di-carbonitrile Polyphenyls
}

\author{
Suetlana Klyatskaya,* Florian Klappenberger,* Uta Schlickum, Dirk Kühne, \\ Matthias Marschall, Joachim Reichert, Régis Decker, Wolfgang Krenner, \\ Giorgio Zoppellaro, Harald Brune, Johannes V. Barth, * and Mario Ruben*
}

This Feature Article reports on the controlled formation and structurefunctionality aspects of vacuum-deposited self-assembled organic and metal-organic networks at metal surfaces using ditopic linear and nonlinear molecular bricks, namely di-carbonitrile polyphenyls. Surface confined supramolecular organization of linear aromatic molecules leads to a fascinating variety of open networks. Moreover, cobalt-directed assembly of the same linear linkers reveals highly regular, open honeycomb networks with tunable pore sizes representing versatile templates for the organization of molecular guests or metal clusters and the control of supramolecular dynamers. In addition, the 2D nanopore organic networks act as arrays of quantum corrals exhibiting confinement of the surface-electronic states of the metallic substrate. A reduction of the linker symmetry leads to the formation of disordered, glassy coordination networks, which represent a structural model for amorphous materials. advanced nanotechnology, presenting exquisite arrangements with atomic or molecular level precision. ${ }^{[2]}$

More recently, well-defined surfaces have been employed as platforms for the fabrication of molecular nanostructures and networks. ${ }^{[3]}$ For example, high-resolution patterning is at the basis of a variety of modern technologies. The rate of advancement of modern semiconductor and data storage devices is closely linked to the ability to create scaled high-resolution patterns on substrates of materials. Therefore, surface patterning is an important issue not only for fundamental research in nanoscale science, but also for several aspects of material science and new functional materials applications, such as the fabrication of light-emitting devices, semiconductor microelectronics, the generation of masks

\section{Introduction}

Supramolecular science and molecular self-assembly are of paramount importance for the development of novel functional materials with molecular-level feature control. There has been tremendous progress in the engineering of highly organised molecular architectures in three dimensions, where noncovalent interactions (e.g., hydrogen bonding, metal coordination, van der Waals forces, $\pi-\pi$ interactions, and/or electrostatic interaction) mediate the expression of structural motifs and stabilise either discrete supramolecules or extended networks. ${ }^{[1]}$ In particular, supramolecular chemistry has become an important method in bulk media. Ordered self-assembled materials have shown enormous potential in a wide variety of fields of and templates, or the production of optical components

Upon the two-dimensional (2D) confinement on a conducting substrate, the realized systems are amenable to direct observations using scanning probe techniques and in-depth scrutiny by powerful space-averaging surface science tools. ${ }^{[4]}$ However, this new situation also requires the exploration of the underlying design principles that provide a unqiue playground for the bottom-up design of matter. ${ }^{[5-7]}$ Herein, we present a review summarizing work with a simple class of aromatic molecules-di-carbonitrile polyphenyls-and their architectures formed at metal surfaces under vacuum conditions. The molecular building blocks have been synthesized with different length and symmetry to extract systematic trends. Firstly, we
Dr. S. Klyatskaya, Dr. F. Klappenberger, D. Kühne, M. Marschall,

J. Reichert, W. Krenner, Prof. J. V. Barth

Physik Department E20

Technische Universität München

D-85748 Garching, Germany

E-mail: svetlana.klyatskaya@kit.edu; fklapp@ph.tum.de; jvb@ph.tum.de

Prof. J. V. Barth

Department of Physics \& Astronomy

University of British Columbia

Vancouver, 2355 East Mall, V6T 1Z4, Vancouver, Canada

\author{
Dr. U. Schlickum, Dr. R. Decker, Prof. H. Brune \\ Institute of Condensed Matter Physics \\ Ecole Polytechnique Fédérale de Lausanne \\ $\mathrm{CH}-1015$, Lausanne, Switzerland \\ Dr. S. Klyatskaya, Dr. G. Zoppellaro, Prof. M. Ruben \\ Institut für Nanotechnologie \\ Karlsruher Institut für Technologie \\ D-76344 Eggenstein-Leopoldshafen, Germany \\ E-mail: mario.ruben@kit.edu \\ Prof. M. Ruben \\ IPCMS-CNRS UMR 7504 \\ Université de Strasbourg \\ 23 Rue du Loess, 67034 Strasbourg, France
}


survey the organisation of purely organic layers, where structures with unexpected complexity occur. Secondly, we assess metallosupramolecular nanostructuring in 2D, exploiting coordination interactions between metal atoms and the carbonitrile ligands. Thereby, we encountered unusual coordination motifs that lead to either regular open honeycomb or disordered networks depending on the linker symmetry. Thirdly, we provide examples where the realized supramolecular layer structures yield new functional properties. They qualify for instance as templates for the organisation of metal clusters, for the confinement of electrons or molecular guests in nanopores, and for the control of supramolecular dynamics.

\subsection{Surface-Confined Self-Assembly}

The self-assembly of atoms and/or molecules on atomically welldefined surfaces under the ultra-high-vacuum (UHV) condition has demonstrated in recent years its high potential for the production of functional systems with nanometer dimensions. ${ }^{[8]}$ The straightforward self-assembly protocol represents the bottom-up approach creating surface patterns and devices on substrates in a controlled periodic and massively parallel manner. ${ }^{[3,9]}$ It is based on the 2D near-surface assembly of organic ligand systems ${ }^{[10]}$ and/or their coordination by metal-ions. ${ }^{[11-13]}$ Here, surfaceassisted self-assembly will be exemplified by following two distinct strategies: i) the formation of supramolecular layers of pure organic molecules by weak bonding schemes and (ii) the metaldirected organization of the same ligands by the formation of coordinative bonds (Scheme 1).

The formation of purely organic supramolecular patterns on metal substrates depends on both, the interactions within the molecular adlayer as well as on the nature of the moleculesubstrate interaction. ${ }^{[14]}$ Hydrogen bonding and dipole-dipole interactions arising from functional groups play an important role in the process. Using molecular self-assembly the desired structure can be programmed by the nature and position of the functional groups in the instructed molecules. Thus, a series of porous molecular networks has been developed consisting of 2D supramolecular structures, using a binary mixture of hydrogenbonding complementary components..$^{[5,15,16]}$ Although the use

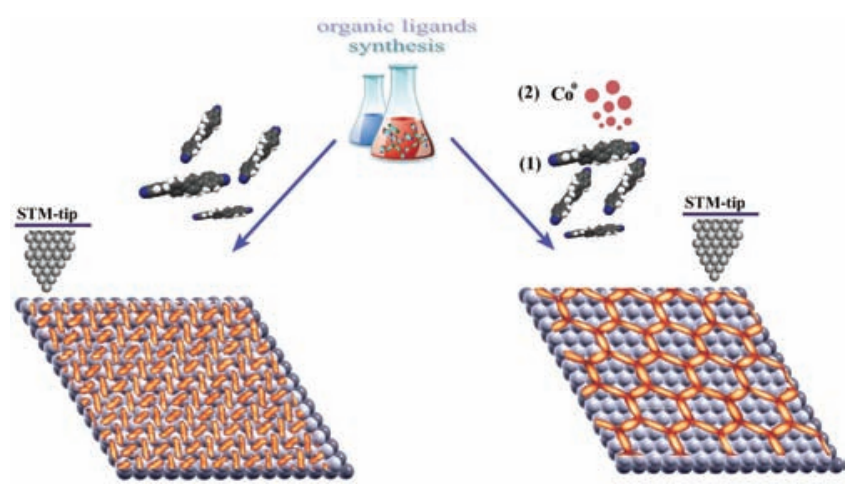

Scheme 1. Schematic representation of the described bottom-up structuring approaches: surface-confined supramolecular organic arrays (left) and metal-directed assembly of coordination networks (right).

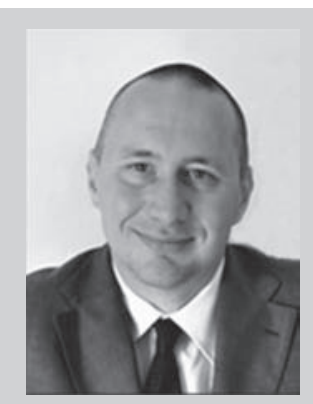

Mario Ruben obtained his PhD in 1998 from the University of Jena, Germany, under supervision of Prof. D. Walther. During a DAAD post-doctoral fellowship he worked in Prof. J.-M. Lehn's research group at the ISIS-ULP in Strasbourg, France. 2001 he moved to the Institute of Nanotechnology in Karlsruhe, where he is currently working as a group leader. In addition, he accepted a position as professor at the Universite de Strasbourg, France, in 2010. His research interests involve the design, synthesis and physical characterization of functional molecule-based systems and their implementation into operational nanosystems. He is the co-ordinator of several European networks dealing with molecular nanodevices.

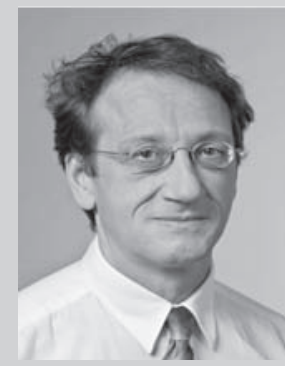

Johannes V. Barth is a Chair in Experimental Physics at the Physik Department, Technische Universität München and Adjunct Professor at the Department of Physics \& Astronomy, The University of British Columbia, Vancouver. His present research interests are mainly in molecular nanoscience and surface chemical physics. He holds a physics degree from Ludwig-Maximilians-Universität München and conducted his PhD studies in Physical Chemistry at the Fritz-Haber-Institut der Max-PlanckGesellschaft, Berlin. He was a postdoctoral fellow at the IBM Almaden Research Center and the Fritz-Haber-Institut, Team Leader and Visiting Professor at Ecole Polytechnique Fédérale de Lausanne, and nominated a Canada Research Chair at UBC, before joining the Faculty of TU München in 2007, where he received an ERC Advanced Grant in 2010.

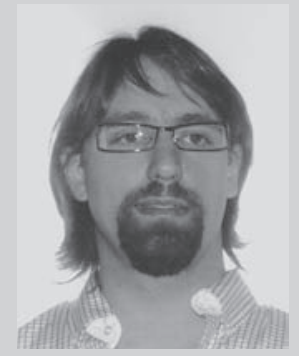

Florian Klappenberger obtained his $\mathrm{PhD}$ in 2004 from the University of Regensburg, Germany, where he investigated electric transport properties of semiconductor diodes under the influence of intense $\mathrm{THz}$ radiation. During his postdoctoral activities at EPF Lausanne, Switzerland, he developed a program focusing on the design and investigation of surface-confined functional molecular nanostructures and organic thin films. The comprehensive physico-chemical characterization is based on a multi-technique approach combining the complementary information of scanning tunnelling microscopy and spectroscopy with synchrotron radiation studies. Since 2007 he is working towards a habilitation at the TU München.

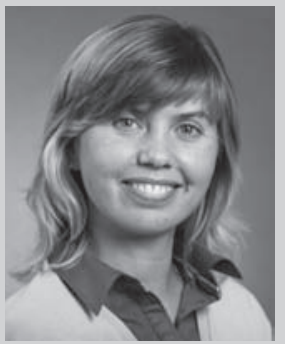

Svetlana Klyatskaya obtained her PhD in 2002 from the Institute of Chemical Kinetics and Combustion, SB RAS, Novosibirsk, Russia. She worked on shape-persistent macrocycles with Professor S. Höger at the University of Karlsruhe. Since 2006, she has occupied a bridging position between the supramolecular engineering of functional nanosystems and organic chemistry groups working in the field of the self-assembly of functional organic molecules at surfaces and molecular magnetism. 
of weak interactions has evident disadvantages, it frequently provides excellent long-range ordering. A binary mixture of melamine and perylene tetracarboxilic diimide has lead so far to an extended hexagonal network. ${ }^{[5]}$ Moreover, a supramolecular structure exhibiting a similar topography was observed in case of anthraquinone molecules as well.[17]

Conceptually, the network stability can be increased by incorporating metal atoms forming strong metal-organic coordination bonds. Hence, a variety of regular 2D metal-ion arrays was so generated just by controlling the codeposition parameters for organic dicarboxylic acid derivatives or pyridyl-containing ligands and iron atoms on $\mathrm{Cu}$ (100), Ag (111) and Ag (100) surfaces under ultrahigh vacuum (UHV)-conditions. ${ }^{[11,13,18]}$ In this way, metallo-supramolecular engineering allowed generation of nearly identical hexagonal networks that were tailored under maintenance of their specific shape, periodicity and symmetry. Thus, our approach combines a straightforward, massively parallel fabrication with an exquisite control over the supramolecular organization parameters (e.g., shape, periodicity, composition, and mesoscale organization) of the nanoscale surface structures formed. Scanning tunneling microscopy (STM) allows us to probe the composition, structure, and orientation of molecules at surfaces at the atomic scale. Complementary to this local probe technique the space-averaging method nearedge X-ray absorption fine-structure spectroscopy (NEXAFS) provides the advantages of element specific information on molecular conformation. ${ }^{[19]}$

\subsection{Materials: Polyphenyls}

The potential advantage of the organic self-assembly bottom-up approach over the conventional, solid state top-down fabrication includes lower processing cost and low-temperature processing with novel implementation environments, e.g., plastic substrates, which may allow a patterning at the atomic scale with atomic resolutions of mechanically rugged and flexible systems. Most of the used compounds are classified as conjugated polyaromatic molecules and belong to p-type semiconductor materials with outstanding optical and electronic properties, such as photoluminescence with emission colors from the near infrared ${ }^{[20]}$ to the ultraviolet ${ }^{[21]}$ spectral range (Scheme 2). The electronic transport through the moleculs is of a great importance, e.g., for electroluminescence, and depends crucially on their mutual spatial arrangement. Organic semiconductor properties are essentially found for two different classes of molecules: conjugated polymers and small-molecule polyaromatic materials. In contrast with conjugated polymers, organic materials based on small molecules often spontaneously arrange into polycrystalline films with charge carrier mobilities compatible at cryogenic temperature with inorganic semiconductor crystals, ${ }^{[22]}$ e.g., sexithiophene, perylenetetracarboxylic-dianhydride, metal-phthalocyanines. ${ }^{[23-26]}$ Oligo(p-phenyl)s (OPPs) are under searchlight after the first light-emitting device based on sexiphenyl could be realized. ${ }^{[28]}$ These devices emit light in the entire visible range and even provide polarized light under the appropriate conditions. Moreover, this class of molecules has been proven to act as useful rigid-rod cores for liquid-crystalline materials ${ }^{[29]}$ and as amphiphilic materials for biological applications. ${ }^{[30]}$ Conceptually, organic compounds provide many advantages compared to inorganic materials, in particular the ability of fine-tuning the properties of the material through functional group manipulation. Genuine oligophenyls are insoluble, thus difficult to deposit in the form of thin films. The introduction of long alkyl side chains in the OPP can improve the solubility ${ }^{[31]}$ in a variety of organic solvents, and thin films can be readily prepared. On the other hand chemical tuning enables fine modification of the optical properties, e.g., polyphenyls with phenolic $\mathrm{OH}$ groups, polyPh $(m) \mathrm{OH}$, exhibit significant solvatochromism. ${ }^{[32]}$ Thus, supramolecular chemistry links organic chemistry to material science and presents one of the fastest growing areas of chemistry research.

\section{Supramolecular Engineering}

The creation of molecular materials that have desired electrical conductance and magnetic properties demands the precise mutual arrangement of the individual molecules. Such

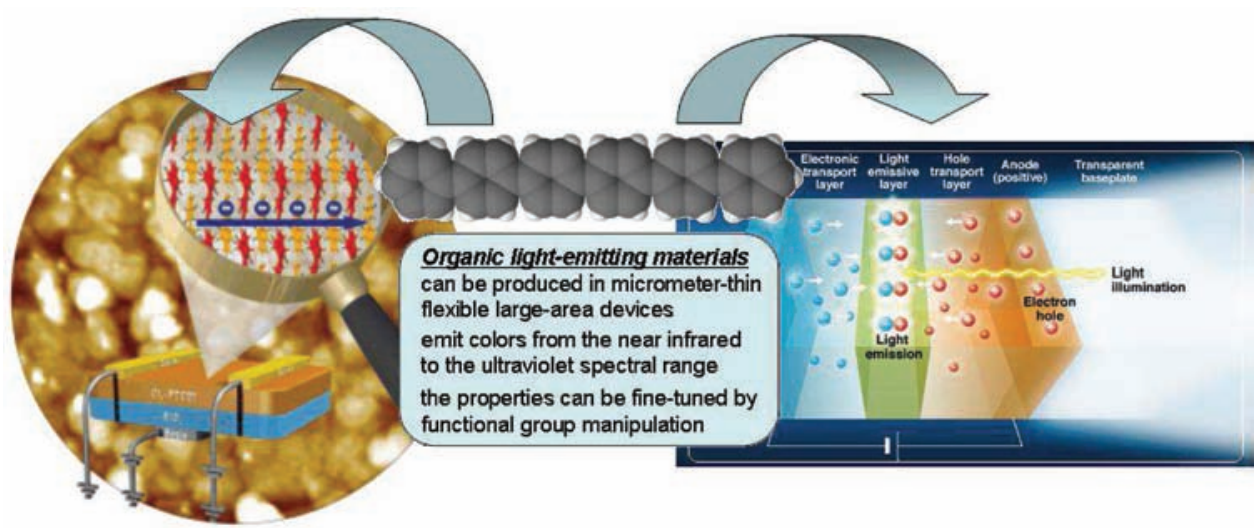

Scheme 2. Schematic representation of great importance of organic p-type semiconductors for technological application. Adapted with permission. ${ }^{[27]}$ 
controlled arrays can be achieved by self-assembly of carefully designed functional molecular building blocks. Even weak but selective noncovalent linkages can program the formation of highly organized systems with molecular-level feature control. Adsorption, mobility, and intra- or intermolecular interactions, all of which depend on the substrate crystalline lattice, chemical nature, and symmetry, are key parameters that govern the formation of molecular architectures. The subtle balance between molecule-molecule and molecule-surface interactions is at the origin of the formation of supramolecular order. The noncovalent design of molecular nanoarchitectures on solid surfaces requires the joined exploration of concepts from both coordination chemistry and mastering the principles of molecular architectonic on well-defined substrates, whereby sophisticated atomic-resolution tunneling microscopy and spectroscopy techniques open up intriguing novel avenues for nanostructured materials design.

\subsection{Submonolayer Coverage}

A systematic study on the engineering of a series of regular organic networks with increasing complexity upon extending the length of the employed linear molecular bricks on $\mathrm{Ag}(111)$ was reported..$^{[33,34]}$ Linear dicarbonitrile-polyphenyl molecular rods, $\mathrm{NC}-\mathrm{Ph}_{n}-\mathrm{CN}$, whereby $n=3,4,5,6$, were investigated. The lengths vary from 1.7 to $2.9 \mathrm{~nm}$ (Figure 1) in $0.4 \mathrm{~nm}$ steps within the number of phenyl groups incorporated. To design these building blocks authors have developed a new synthesis based on Suzuki coupling scheme of corresponding bis-iodo-polyphenyls

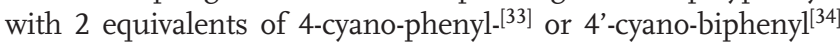
boronic acids in the presence of catalytic amount of $\operatorname{Pd}(0)$.

The molecules were deposited onto a clean Ag(111) surface kept at $300 \mathrm{~K}$ from a quartz crucible in an organic molecular beam epitaxy source (OMBE). For imaging the samples were cooled down to $10 \mathrm{~K}$. All STM images presented have been taken at $10 \mathrm{~K}$, except where noted differently. The molecularlevel STM data reproduced in Figure 1 show a series of 2D supramolecular networks formed upon the deposition of the molecular building blocks on atomically flat and clean $\mathrm{Ag}(111)$ terraces. Single rodlike protrusions in STM topographs correspond to individual molecules.

Depending on the length of the employed molecules a varying degree of network complexity becomes apparent. The smallest species (NC- $\left.\mathrm{Ph}_{3}-\mathrm{CN}\right)$ forms a densely packed chevron pattern (Figure 1a) with molecules lying only along two different crystallographic directions within a given domain. The situation becomes more complicated in the case of the organic networks formed by $\mathrm{NC}-\mathrm{Ph}_{4}-\mathrm{CN}$ molecules. The longer aromatic molecular backbone triggers a spreading into an open geometry comprising chiral rhombic cavities (Figure 1b). It was found that in both cases all $\mathrm{N}$-atoms lie on identical sites on the subjacent $\mathrm{Ag}(111)$ lattice. In these arrangements, both networks are commensurate with the underlying surface, and in both patterns, half of the molecules lie along the [ $1-10]$ direction, while the other

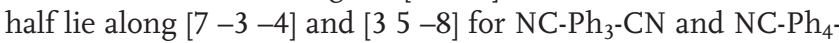
$\mathrm{CN}$, respectively. Unsubstituted quaterphenyl and sexiphenyl molecules with a weak intermolecular interaction in the submonolayer coverage also self-assemble along this high-symmetry axis on $\mathrm{Au}(111)$ and $\mathrm{Ag}(111)$ surfaces, respectively. ${ }^{[35]}$

When comparing the assemblies formed by quinquephenyl and sexiphenyl building blocks $\left(\mathrm{NC}-\mathrm{Ph}_{5}-\mathrm{CN}, \mathrm{NC}^{-} \mathrm{Ph}_{6}-\mathrm{CN}\right)$ an even more complex nanoporous chiral kagomé lattice evolves (Figure 1c,d). Moreover, sexiphenyl-dicarbonitrile patterned the $\mathrm{Ag}(111)$ surface with different types of nanoporous networks (Figure 1d) coexisting simultaneously over square-micrometer wide areas and separated only by step edges. ${ }^{[34]}$ The ends of four molecules join together forming nodal bonding motifs similar for all nanoporous networks, although not equal, in contrast to the densely packed chevron pattern, where nodes cannot be defined. In spite of the nodes similarity, the molecular end groups in the quinquephenyl kagomé networks come much closer than in the nodal structure of simpler lattices such as the rhombic quaterphenyl case or the rectangular phases formed by sexiphenyl derivative. Consequently, a flat bonding of the terminal benzonitrile groups can be excluded; rather they exhibit a significant rotation (about $20^{\circ}$ ) around the long molecular axis. In contrast, $\mathrm{NC}-\mathrm{Ph}_{3}-\mathrm{CN}$ molecules in the densely packed chevron pattern adopt a flat alignment of the terminal benzonitrile groups on the surface. ${ }^{[33]}$ A detailed discussion of the epitaxy of different networks formed by $\mathrm{NC}-\mathrm{Ph}_{6}-\mathrm{CN}$ on $\operatorname{Ag}(111)$ was recently described. ${ }^{[34]}$ The data evidence that in the rectangular phase molecules are aligned in the crystallographic $\left\langle\begin{array}{lll}-1 & 1 & 0\rangle\end{array}\right.$ and $\langle-1-12\rangle$ directions. With a different tip state with good lateral resolution for intramolecular features, the molecules oriented in the two directions are imaged differently. ${ }^{[34]}$ Whereas the molecules in the $\left[\begin{array}{lll}-1 & 1 & 0\end{array}\right]$ direction appear with a zigzag contour, the ones in the $\left[\begin{array}{lll}-1 & -1 & 2\end{array}\right]$ direction show a shape
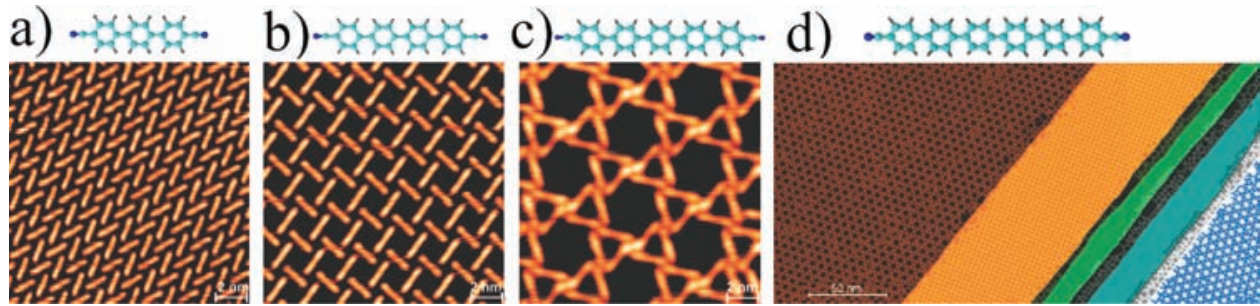

Figure 1. Steering network complexity with the extension of the molecules self-assembled on the $A g(111)$ surface. a) Densely packed chevron layer formed by the NC-Ph $-\mathrm{CN}$ species. b) NC-Ph${ }_{4}-\mathrm{CN}$ molecules assemble in an open rhombic network. c) Kagomé lattice formed by $\mathrm{NC}-\mathrm{Ph}_{5}-\mathrm{CN}$ molecules, comprising four-fold nodes and quasi-hexagonal and trigonal cavities. d) The scanning tunnelling microscopy (STM) topography of NC-Ph ${ }_{6}-\mathrm{CN}$ molecules shows the coexistence of different regular networks. Structure models of molecular building blocks are depicted above the STM images. Their respective lengths are 1.66, 2.09, 2.53 and $2.96 \mathrm{~nm}$ (light blue, C; dark blue, N; gray, H). Adapted with permission. ${ }^{[33,34]}$ Copyright 2008, American Chemical Society and 2009, American Chemical Society, respectively. 

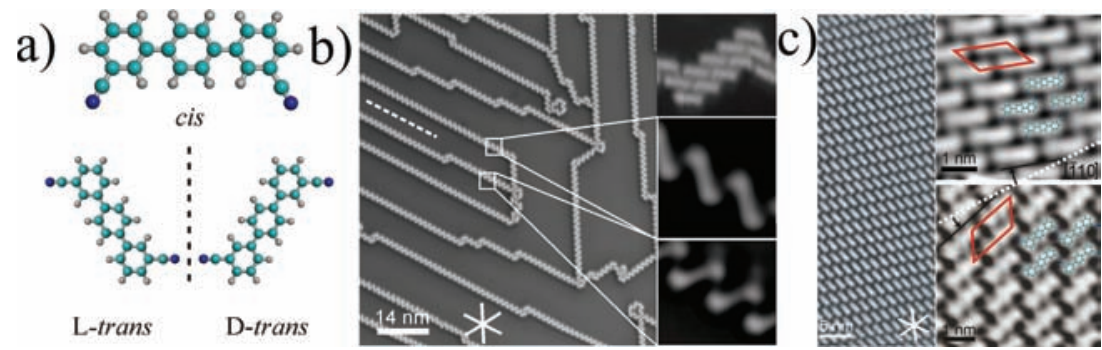

Figure 2. STM overview of a molecular island with a molecular model of $m \mathrm{NC}-\mathrm{Ph}_{3}-m \mathrm{~m}$ molecule (light blue, C; dark blue, N; gray, H). a) The cis-(top), L- and mirror-symmetric D-trans (bottom) arrangements. b) High-resolution STM image of linker molecules assembled in linear structures. Scale bar $14 \mathrm{~nm}$ (left). The magnified views demonstrate the chirality of the nanochains (middle and lower inset) and the transition to 2D island formation (upper inset). c) High-resolution STM images of a densely packed highly ordered molecular island on the $\mathrm{Ag}(111)$ substrate composed of L-trans molecules (top) and D-trans molecules (bottom). Adapted with permission. ${ }^{[36]}$

symmetric to their long molecular axis. A similar zigzag contour has been reported for sexiphenyl on $\mathrm{Ag}(111) .{ }^{[35]}$ There, the authors showed that the edge of a phenyl ring appears bright if the center of the corresponding off-axis $\mathrm{C}-\mathrm{C}$ bond is located on top of a silver surface atom; if the center of a C-C bond is positioned over a bridge site, the corresponding phenyl ring edge appears darker. The similar alignment of sexiphenyl and NC- $\mathrm{Ph}_{6}$-CN in $\left\langle\begin{array}{llll}1 & 0\rangle\end{array}\right.$ implies that the aromatic backbone plays an important role for the epitaxy.

All nodal motifs, and thus the networks based on them, exhibited chirality, originating from the supramolecular organization of achiral molecules $\mathrm{NC}-\mathrm{Ph}_{n}-\mathrm{CN}(n=4-6)$ on an achiral surface. The chirality is introduced by the polar nature of $\mathrm{CN}$ endgroups, preventing a straight orientation toward the node center. ${ }^{[3,34]}$ The molecules have an equal probability to assemble into the one or other enantiomorphic arrangement as found in segregated enantiomorphic domains.

The highly symmetric ditopic linkers discussed so far display a unique number of bonding motifs within the formed structures leading to extended, regular supramolecular arrangements.

In contrast, the bent, nonlinear linkers are prochiral and thus can display a multiplicity of distinct network elements due to the symmetry-breaking effect of the surface. Using a nonlinear linker featuring an increased conformational phase space due to the positioning of the $\mathrm{CN}$ moieties at meta positions $\left(m \mathrm{NC}-\mathrm{Ph}_{3}-m \mathrm{CN}\right)$ (Figure 2a) the supramolecular organization and layer formation of the prochiral molecule absorbed on the Ag(111) surface was investigated. ${ }^{[36]}$

Following room temperature deposition, molecules adopt a nearly flat adsorption geometry. Moreover, the generation of two-dimensional confinement projects the large conformational space of the molecular library into three stereoisomers, namely two mirror-symmetric trans- and one cis-species. At room temperature and for submonolayer coverages on both, the $\mathrm{Ag}(111)$ and $\mathrm{Cu}(111)$ surfaces, isolated molecules exhibit a high diffusivity and conformational freedom. Therefore, the molecular surface fluid consists of equal proportions of cis- and trans-species. Upon cooling down to $6 \mathrm{~K}$ extended supramolecular ribbons have evolved (Figure $2 \mathrm{~b}$ ) showing enantiormorphic characteristics of the molecular packing schemes. Thus, the self-assembly process during condensation upon thermal quenching is a chiroselective process. The trans-conformation is revealed to be more favorable here.

At higher coverage close-packed islands are built by the dicarbonitrile linkers occuring in their trans-conformation (Figure 2c). These islands appear in a total of six domains formed by two enantiopure variants for each of the three high-symmetry directions of the crystal.

In all cases the supramolecular organization is stabilized by attractive and directional, noncovalent interactions of molecules with the next neighbors via the carbonitrile end groups developing two non-covalent bonds with next-neighbor phenyl moieties. The ordering within the expressed supramolecular arrays reflects a substrate templating effect, steric constraints and the operation of weak lateral interactions mainly originating from the carbonitrile endgroups. These results indicate that the 2D layer formation of these molecules reflects a special 2D chirality scenario that has not been described so far. It is, thus, masterly demonstrated that unique bonding motifs and supramolecular surface patterns can be achieved with adsorbed simple molecular units; i.e., surface-confined self-assembly protocols using tailored adaptive molecular species open the way to engineer complex hierarchic nanoarchitectures, which set the base for further functionalization.

\subsection{Saturated Monolayer Coverage}

Properties of organic (opto)electronic devices depend strongly on the degree of ordering in the active layer. ${ }^{[8]}$ Therefore, governing the order in organic films may allow to steer the properties of the resulting applications. Thus, the conformation and orientation properties of dicarbonitrile-oligophenyl molecules $\left(\mathrm{NC}-\mathrm{Ph}_{6}-\mathrm{CN}\right.$, Figure 1) were studied in thin films of varying thickness grown on the Ag (111) surface (Figure 3). In contrast with submonolayer coverages discussed above, approaching
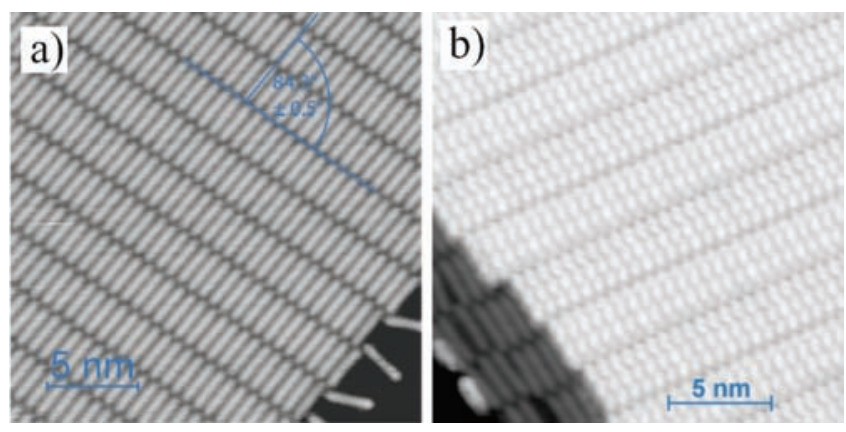

Figure 3. Comparison between ordering in thin films of $\mathrm{NC}-\mathrm{Ph}_{6}-\mathrm{CN}$ samples with different coverage. a) Densely packed phase at a saturated monolayer (1 ML coverage). b) STM image of a sample with 1.5 monolayers. Molecules in the first layer appear as sticklike protrusions, while for molecules in the second layer a zigzag-structure can be identified. Adapted with permission. ${ }^{[34]}$ Copyright 2009, American Chemical Society. 
monolayer saturation, a single dense-packed phase forms exhibiting a well-defined ordering with all molecules in a parallel alignment (Figure 3a).

When even more molecules are added, they do not edge into the dense-packed molecular monolayer as in the case of sexiphenyl molecules, ${ }^{[3]}$ but form a second layer on top. A sample with approximately 1.5 monolayers (MLs) of $\mathrm{NC}-\mathrm{Ph}_{6}$-CN molecules (Figure 3b) demonstrates different appearance depending on the layer. Molecules in the first layer appear as sticklike fea-
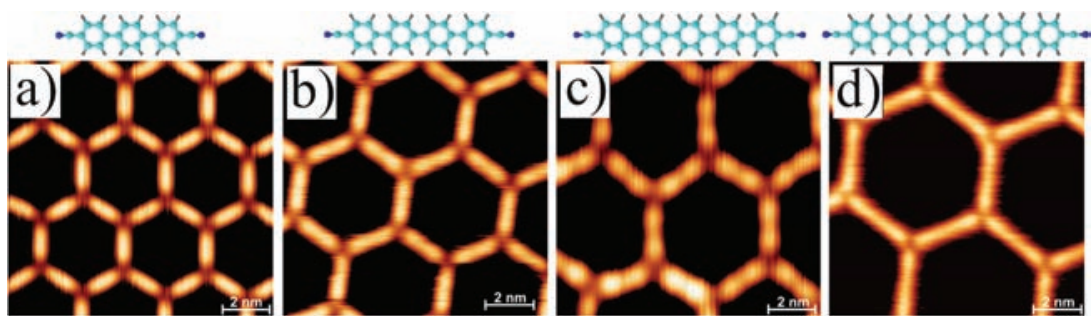

Figure 4. STM topographic data of the metal-organic nanomesh formed by Co-directed assembly of a) $\mathrm{NC}-\mathrm{Ph}_{3}-\mathrm{CN}$, b) $\mathrm{NC}-\mathrm{Ph}_{4}-\mathrm{CN}$, c) $\mathrm{NC}-\mathrm{Ph}_{5}-\mathrm{CN}$, and d) $\mathrm{NC}-\mathrm{Ph}_{6}-\mathrm{CN}$, respectively. Adapted with permission. ${ }^{[43,44]}$ Copyright 2007, American Chemical Society and 2009, American Chemical Society, respectively.

\section{tures similar to the appearance in Figure 3a.}

The molecules in the second layer are characterized by distinct intra-molecular features producing a zigzag line of bright structures with a face-on fashion of molecular orientation for all molecules. An evaluation of STM images for a large number of different approaches suggests, that a third layer was not present on that sample. Since the interaction between the first and the second layer of physisorbed molecules are nearly the same, the data indicates a layer-by-layer growth (Franck van der Merwe type). If Stranski-Krastanov growth took place, STM images with three or more layers of molecules should have been found at least occasionally.

The molecular conformation for the whole series of molecules was studied with NEXAFS for different coverage regimes. ${ }^{[38]}$ The spectroscopic data reveals that the molecular conformation is always characterized by a twist angle $\gamma$ between adjacent phenyl rings with varying values between $20^{\circ}$ to $50^{\circ}$ depending on the film thickness. For molecular coverage less than half a monolayer $\gamma$ is typically around $20^{\circ}$ to $30^{\circ}$ and increases with the coverage reaching approximately $50^{\circ}$ for a full monolayer. For thin films with a thickness of several (up to 20) monolayers $\gamma$ stays constant at the same value as for the monolayer case. Furthermore, the NEXAFS data indicate, that the average molecular plane, out of which adjacent phenyl rings are tilted up respectively down by $\gamma / 2$, is oriented parallel to the substrate for all cases. Thus, the lateral hydrogen bonds between molecules in the open porous networks lead to a planarization of the phenyl rings compared to the parallel-aligned saturated monolayers without the lateral hydrogen bonds.

The combined STM and NEXAFS ${ }^{[38]}$ data lead to the following conclusion: the well-defined first monolayers act as a high-quality template forcing $\pi$-stacked growth of the following layers. The addition of the NC group to oligophenyls avoids the oligophenyl-typical herringbone structure ${ }^{[39]}$ and thus improves the molecular ordering. This method may offer a novel way to increase the performance of future devices based on extended oligophenyls.

\section{Metal-Directed Assembly}

Chemists are proficient at directing the synthesis of small molecules, but fine-tuning the structures of supramolecular networks is far more taxing. In this respect, metallosupramolecular engineering gives access to a variety of grid structures based on the coordination of organic linkers with metal centers. ${ }^{[40]}$ In an elegant way the fabrication of surface-supported highly regular metal-organic nanomeshes covering terraces over $\mu \mathrm{m}^{2}$ large areas as single domains was recently demonstrated exploiting the high directionality of organometallic coordination of the carbonitrile groups $(\mathrm{CN})^{[41]}$ in ditopic linear polyphenyl linkers.

\subsection{Ditopic Linear Polyphenyl Linkers}

Subsequent exposure of the molecular films discussed above to a beam of Co atoms at room temperature leads to the assembly of metal-organic networks. The STM data reproduced in Figure 4 demonstrates that cobalt centers with preadsorbed linker molecules of different lengths form a series of open nanomeshes with a tunable cavity size on the $\mathrm{Ag}(111)$ substrate. Independent on the number of backbone phenyl rings highly regular arrays comprising nanometer-sized honeycomb cavities are formed. The area of the enclosed hexagons increases with the number of phenyl rings incorporated in the molecular linkers' backbone. ${ }^{[42-44]}$ Accordingly, the cell size expands stepwise from about 10 via 15,20 up to $29 \mathrm{~nm}^{2}$ for $n=3,4,5$, and 6 , respectively.

The nanoporous honeycomb structure features an unusual but robust threefold coordination of $\mathrm{CN}$ moieties to cobalt centers in the ideal network with a metal-linker stoichiometry of 2:3. ${ }^{[2-44]}$ As shown for the para-sexiphenyl-dicarbonitrile linkers ${ }^{[4]}$ an epitaxial fit (Figure 5a) between honeycomb and atomic $\mathrm{Ag}(111)$ lattice promotes layers with excellent regularity.

All nanomeshes comprise the same threefold coordination motif with Co atoms positioned at the nodes. The Co centers connect the molecules symmetrically resulting in an angle of $120^{\circ}$ between adjacent ligands.

The threefold coordination of the Co centers in the dicarbonitrile nanomeshes described above could be rationalized with the help of DFT calculations. For the modeling, the molecular linkers were simplified as $\mathrm{NC}-\mathrm{Ph}_{1}-\mathrm{CN}$ retaining the carbonitrile endgroups that interact with the Co centers. In addition to that, the molecules were confined in a plane in accordance with the STM data, showing that the aromatic polyphenyl linkers are adligands. Accordingly, an appreciable electronic hybridization between the Co and Ag atoms occurs, as visualized in Figure 5b.

To date, the obtained networks based on dicarbonitrile coordination of Co centers employing the smooth Ag (111) substrate are of the highest structural quality; they are stable at room temperature and extend over $\mu \mathrm{m}^{2}$ large areas as single domains. 
a)

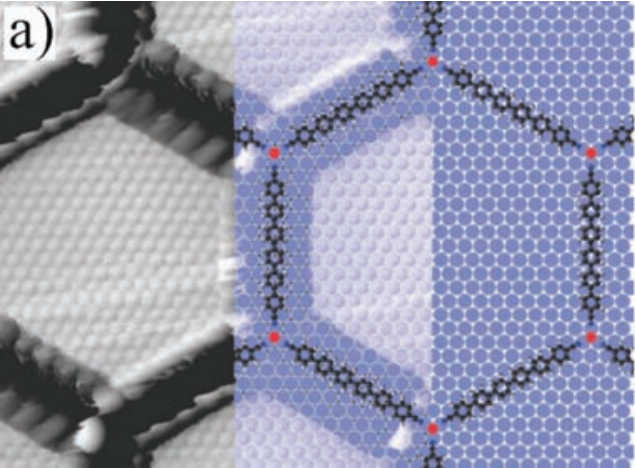

b)
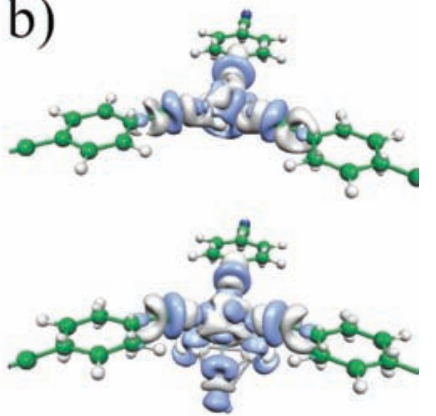

Figure 5. The threefold Co-carbonitrile coordination motif addressed by STM and DFT calculations. a) Left: STM image, showing atomically resolved $\mathrm{Ag}(111)$ surface lattice within the nanocavities of the Co-sexiphenyl-dicarbonitrile nanomesh. Right: a corresponding ball model partly superimposed on the tunneling image. The ball model visualizes the perfect registry between network and substrate. b) Plots of the induced charge density around the cobalt atom in threefold coordination of a model compound (NC-Ph ${ }_{1}-\mathrm{CN}$ ) without (upper) and with (lower) the presence of an $\mathrm{Ag}_{4}$ cluster underneath the transition metal center. The image displays the electron density redistribution around the $\mathrm{Co}$ atom due to the bond formation with the ligands and the $\mathrm{Ag}_{4}$ cluster. Light blue color means charge depletion, and light gray charge accumulation $\left(0.002 \mathrm{e} / \AA^{3}\right)$. Adapted with permission. ${ }^{[43,44]}$ Copyright 2009 , American Chemical Society and 2007, American Chemical Society, respectively.

When deposition procedures are used under Co deficiency, starlike Co-linker motifs with a sixfold coordination of $\mathrm{CN}$ groups to a single Co center were identified. Furthermore, by imposing space limitations in the metal-organic network the formation of fully reticulated disordered networks with coordination numbers varying between three and six were realized. Local deviations from the preferred threefold coordination with characteristic defects were also found when kinetic limitations interfere in the nanomesh formation. ${ }^{[45]}$

\subsection{Ditopic Nonlinear Polyphenyl Linkers}

All metal-organic assembly units described so far rely on linear highly symmetric ditopic linkers, thus only a small number of bonding motifs within the formed structures was revealed. However, a more complex scenario stands when the nonlinear prochiral linkers are considered. As the symmetry of the molecule decreases, the number of conformation states accessible range order. By means of a statistical analysis of the random coordination networks it was determined that the natural ratio of the species present in the 2D surface gas is approximately conserved $(\approx 60 \%$ cis and $\approx 20 \%$ for each trans species). The data clearly reveal the coexistence of nodal motifs with three- or fourfold coordination of cobalt centers by carbonitrile ligands. The encountered coordination nodes were separated into bifurcation motifs and chain motifs based on a scenario mediated by reversible metal-ligand interactions. The predominant bifurcation motifs are of the fourfold cis-cis-Co-trans-trans and the threefold Co-trans-trans-trans node families (Figure 6b,c). The existence of chain motifs is identified readily in Figure $6 \mathrm{~d}$. The predominant chain-forming coordination nodes are of the families cis-cis-Co-cis-cis and cis-cis-Co-cis (modeled in Figure 6e). The proportion of threefold-coordinated nodes in a given network is typically significantly higher than that of fourfold-coordinated nodes. Such observation is in agreement with density functional theory calculations where an energetic preference

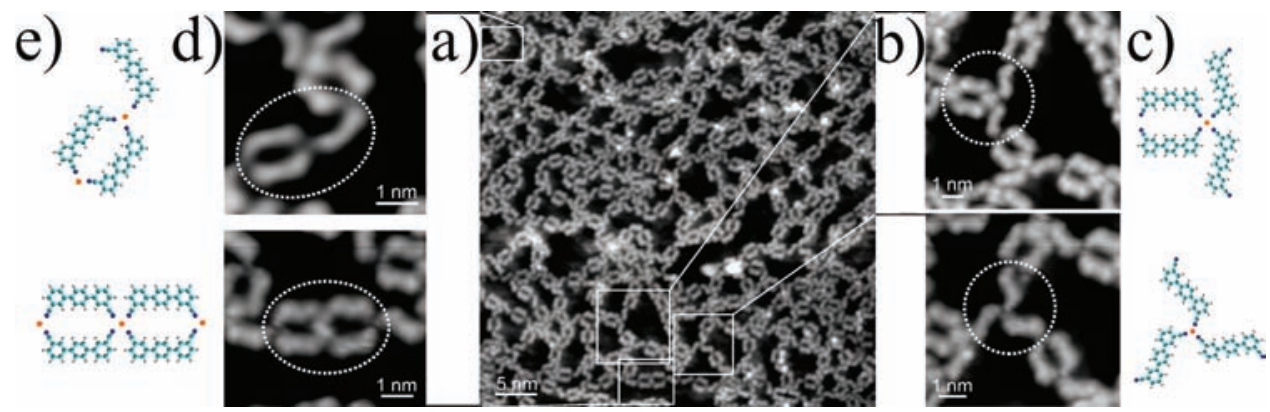

Figure 6. STM overview of the glassy metal-organic coordination network. a) Random coordination string network on $\mathrm{Ag}$ (111) including coordination motifs that underlie the random network with coexisting three- and fourfold coordination nodes. b) Magnified views showing the two dominant bifurcation motifs (cis-cis-Co-trans-trans, upper panel; Co-trans-trans-trans, lower panel) with their corresponding models (c). d) Magnified chain motifs with three (cis-cis-Co-cis, upper panel) and fourfold (cis-cis-Co-cis-cis, lower panel) coordinated cobalt with their corresponding models (e). Light blue spheres: C; dark blue spheres: N; white spheres: $\mathrm{H}$; orange spheres: Co. Adapted with permission. ${ }^{[47]}$ Copyright 2010, Nature Publishing Group. 
for threefold coordination of cobalt centers by carbonitrile functional groups on noble metal surfaces has been found. ${ }^{[4]}$

This work undoubtedly lifted the veil of mystery of structural details of amorphous materials at the molecular level hindered by the lack of long-range order in the bulk. Moreover, it presents a new route towards a distinct class of robust, adaptive compounds and network topologies situated between so-called metal-organic frameworks and covalent polymers.

\section{Functional Nanosystems}

The controlled substrate composition and functionality is of major interest of the many possible applications in nanoscience and nanotechnology. ${ }^{[48]}$ Self-assembly can provide well-organized structures with atomic or molecular level precision. Therefore, molecules are the favourite building blocks to decorate, structure, and functionalise surfaces on the nanometer scale between 1 and $10 \mathrm{~nm} \cdot{ }^{[49]}$

Ordered organic and metal-organic networks on surfaces demonstrate a great potential as templates for the ordered absorption of organic molecules. ${ }^{[5,11]}$ They can be employed as (i) host cavities for guest molecules, (ii) a guide to steer nucleation of metal atoms ${ }^{[50]}$ by offering nucleation sites at their rims ${ }^{[7,51]}$ or nodes, ${ }^{[52]}$ and (iii) templates for the control of supramolecular dynamics. In addition, the room-temperature stability is an important aspect in view of the potential applications of 2D coordination networks.

\subsection{Templating and Steering Molecular Organization}

The appreciable thermal stability and overall robustness ${ }^{[42-44]}$ of the metal-organic honeycomb nanomeshes verified by repeated heating from 10 to $300 \mathrm{~K}$ makes them ideal templates for the (selective) adsorption of guest atoms or/and molecules and their templating on the surface. The open 2D coordination network constructed from NC-Ph${ }_{4}-\mathrm{CN}$ linkers was used to steer the guest molecules trapped inside of the cavities (Figure 7a,b) qualifying them as templates for the spatial organization of nanoscale objects in well-defined environments.

Longer dicarbonitrile $\mathrm{NC}-\mathrm{Ph}_{5}-\mathrm{CN}$ linkers confined in the cavities formed by $\left[\left(\mathrm{NC}-\mathrm{Ph}_{4}-\mathrm{CN}\right)_{3 / 2} \mathrm{Co}\right]_{n}$ nanomeshes are arranged in specific configurations (Figure 7a,b). By increasing the tunnel current from 0.1 to $0.4 \mathrm{nA}$, the molecules can be laterally displaced from one configuration to another.

In case of disordered coordination network (Figure 6a) molecular manipulation experiments ${ }^{[47]}$ were carried out. By a lateral STM tip movement across a chain-terminating molecule it was demonstrated that the terminal molecule can flip during the manipulation procedure without breaking the coordinative bond.

Moreover, the honeycomb nanomeshes qualify as templates to steer the formation of metal nanostructures. Surprisingly, Fe and Co atoms assemble in a similar manner forming the metal clusters exclusively on top of the metal-organic network (Figure 7c,d). Furthermore, for $\mathrm{Fe}$ it was unambiguously revealed that the ligands are the preferential nucleation sites of the networks for temperatures in the range $90-120 \mathrm{~K}$ and the network nodes for temperatures in the range $190-220 \mathrm{~K} \cdot{ }^{[53]}$ Metallic nanostructures have attracted interest since they exhibit magnetic ${ }^{[54]}$ and catalytic properties ${ }^{[55]}$ which often show a strong size dependence. In this respect, size-controlled self-organized growth of metals on template surfaces demonstrated is a promising route to create ensembles of nanostructures with well-defined sizes and a high degree of order over micrometer distances.

\subsection{Supramolecular Dynamics}

The design, synthesis, and operation of multicomponent molecular systems capable of performing specific, directional mechanical movements on the surfaces constitute a fascinating challenge in the field of nanoscience. ${ }^{[56]}$ Using a tailored nanoporous open 2D coordination network constructed from $\mathrm{NC}-\mathrm{Ph}_{6}-\mathrm{CN}$ linkers as a template, it is possible to develop a hierarchic organization to generate collective motion and assembly (Figure 8a), which can be directly monitored at the single-molecule level. ${ }^{[57]}$

Excess of the linker molecules are captured in the network cavities at about $145 \mathrm{~K}$ due to the energy barrier to migrate across the network rims. Moreover, the confined molecules are free to arrange within the pore in energetically favourable configurations and the confined monomers are always attached to the network rim in a specific geometry, reflecting a surface templating effect and weak attractions between phenyl hydrogen and CN endgroups. We observed self-assembly of the free linkers into trimeric arrangement with a threefold nodal bonding motif at the center. Furthermore, STM images
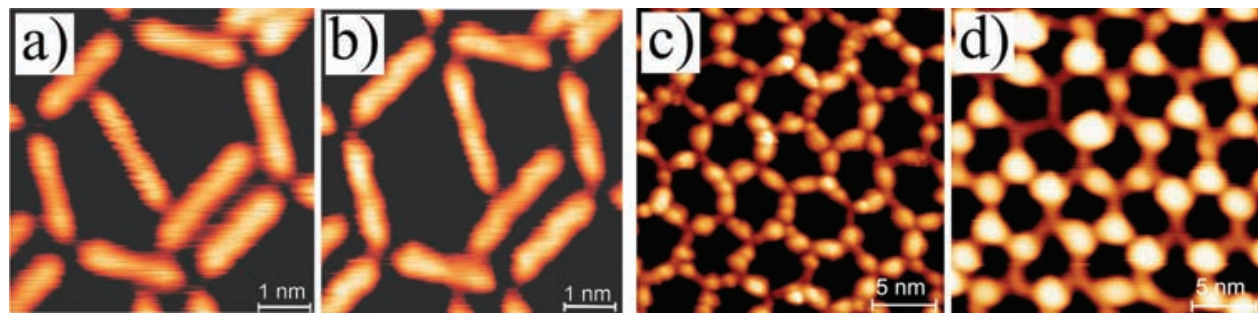

Figure 7. Nanocavities for the host-guest confinement and steering of the self-organized growth of metal nanoclusters. a,b) A template formed by NC-Ph $-\mathrm{CN}$ linkers is used to host two NC-Ph $-\mathrm{CN}$ guest molecules. c,d) Constant current STM image of the Co and Fe decorated (NC-Ph ${ }_{5}$ $\mathrm{CN})_{3} \mathrm{Co}_{2}$ network respectively. Adapted with permission. ${ }^{[43,53]}$ Copyright 2007, American Chemical Society and 2008, American Institute of Physics, respectively. 

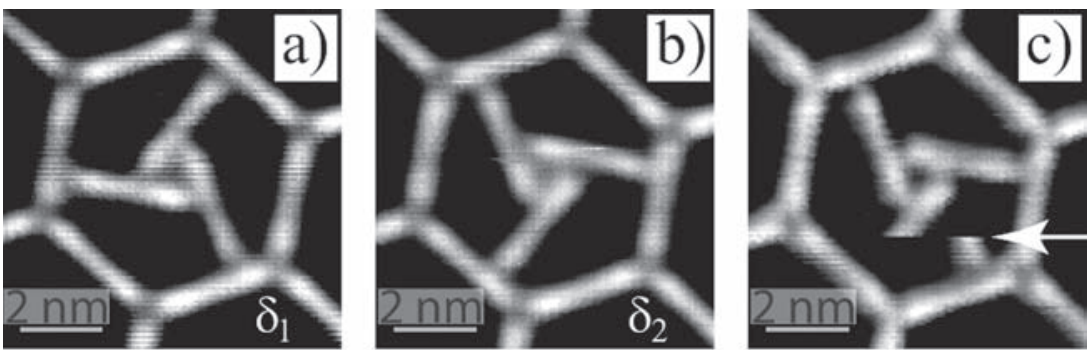

Figure 8. Rotational motion of the caged supramolecular dynamer. a,b) $60^{\circ}$ orientationa switching between a stable $\delta_{1}$ and $\delta_{2}$ configuration with conservation of the chirality signature; images recorded with a time lap of $204 \mathrm{~s}\left(V_{\mathrm{B}}=1.0 \mathrm{~V}, I_{\mathrm{T}}=0.05 \mathrm{nA}, T_{\text {Sample }}=64.4 \mathrm{~K}\right)$. c) Orientation switching event while recording the topography at the position indicated with an arrow; the molecular units are exclusively imaged with positions reflecting preferred configurations $\left(V_{B}=\right.$ $-1.0 \mathrm{~V}, I_{\mathrm{T}}=0.05 \mathrm{nA}, T_{\text {Sample }}=64.8 \mathrm{~K}$ ). Adapted with permission. ${ }^{[57]}$ Copyright 2010, National Academy of Sciences.

evidence that there are two mirror-symmetric configurations of the supramolecular trimer reflecting an organizational chirality. ${ }^{[57]}$

The response of caged trimers to thermal excitations was probed by systematic STM measurements with increasing temperatures. The thermal rotary motions of the guest unit are visualized by the data series in Figure $8 \mathrm{a}-\mathrm{c}$ concentrating on a single caged $\delta$-rotator. An orientational switching event occurred in the time interval between the recording of the images (a) and (b) and concerned the entire supramolecular unit. The possible pathway of disassembly, fast hopping of single molecules and subsequent reassembly into the trimeric unit can be excluded by the conservation of the chirality signature through a large number of rotational motion events. As a consequence, the supramolecular guest must rotate as a concerted unit. At higher temperatures, moreover, a constitutional dynamic of the caged supramolecular entities occurs. The bottom-up construction of caged supramolecular dynamers reveals the remarkable potential of surface-confined
www.MaterialsViews.com

self-assembled nanoporous architectures by exploiting massively parallel fabrication concepts to control dynamic phenomena of caged species at the supramolecular level.

\subsection{Electron Confinement: Quantum Corrals}

Confinement at the nanometer scale can reveal the quantum properties of surface state electrons and may eventually lead to corresponding changes of the electronic properties of the surface. The concept of confinement of electronic surface states applies down to very small, nanoscopic structures ${ }^{[58]}$ playing a crucial role in the properties, function, and performance of advanced materials and in nanometer-scale technologies. The STM has provided new opportunities for investigating these states and their interactions with adsorbates enabling direct imaging in real space. ${ }^{[58]}$ So far, the confinement of surface-state electrons has been demonstrated on single absorbed atoms ${ }^{[51]}$ or rather simple selfassembled molecular structures. ${ }^{[12]}$ But it was shown recently, that complex supramolecular engineering can be used in the design of surface state quantum confinement. ${ }^{[14,59]}$

The open kagomé superlattice (Figure 9a, top) created by the self-assembly of $\mathrm{CN}-\mathrm{Ph}_{6}$-NC molecules on the $\mathrm{Ag}(111)$ tessellate the surface into pores with different size and symmetry. The presence of the organic network is periodically modulating the local density of states and thus creating quasihexagonal and triangular-terminated areas with a distinct electronic structure in each type of pore. Thus, the network acts as a dichotomous array of quantum corrals (Figure 9b, bottom). An inspection of the differential conductance
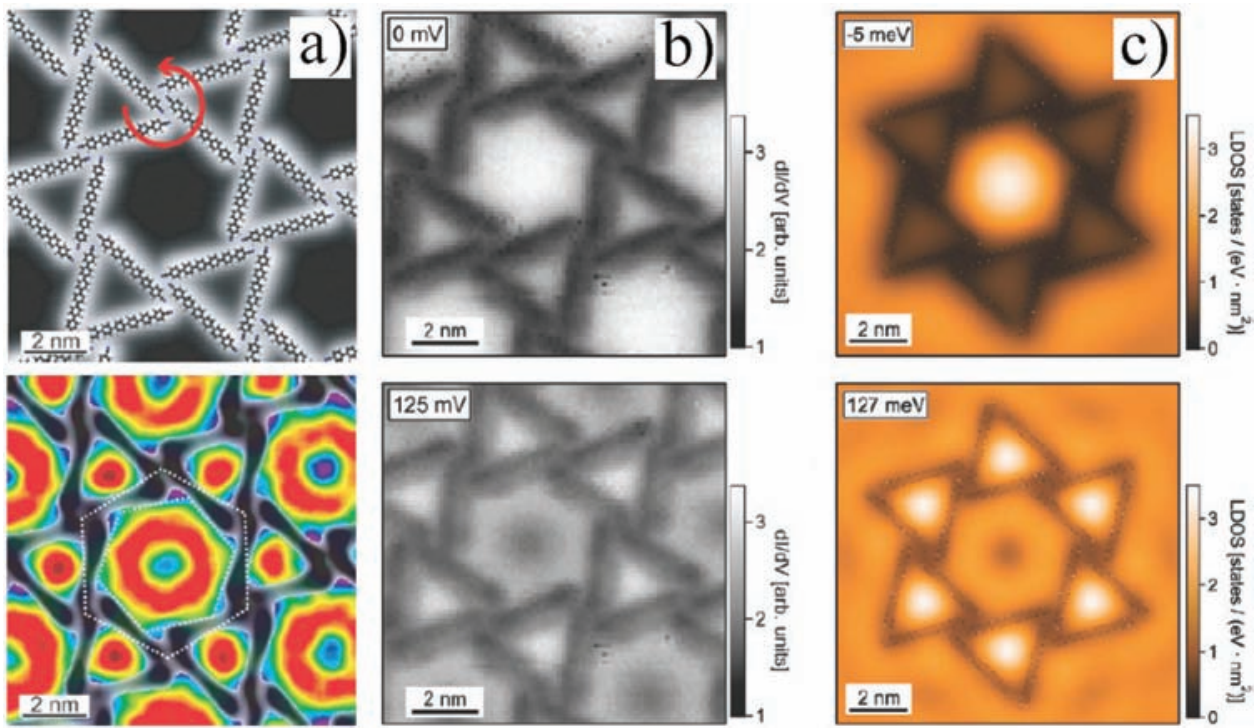

Figure 9. The confinement of surface-state electrons by a complex supramolecular network. a) High-resolution image of the kagomé structure with superimposed ball-and-stick models of the molecules (top) and constant-current $d / / d V$ map (rainbow color coded) simultaneously taken while scanning the topography (bottom). b) Experimental maps (acquired by a set of open feedback loop spectra) of the kagomé network induced standing wave patterns for different bias. c) Calculated LDOS distributions. Adapted with permission. ${ }^{[59]}$ Copyright 2009, American Chemical Society. 
maps at bias voltages $\left(V_{\mathrm{B}}\right)$ from 0 till $500 \mathrm{mV}$ from a set of $84 \times 84$ open feedback loop spectra reveals that at $V_{\mathrm{B}}=0$, a bright domelike structure dominates in the center of the hexagonal pore, while underneath the molecules the electronic density is strongly reduced (Figure 9b, top). Upon increase $V_{\mathrm{B}}$ to $125 \mathrm{mV}$, the intensity distribution in the quasi-hexagon exhibits a minimum in the center, whereas the triangular pores exhibit even brighter intensity than the large pores (Figure 9a, bottom). Besides that, using a scalar version of the electromagnetic boundary element method (BEM), the energy-dependent local density of states, LDOS(E), has been calculated. The simulated standing electron wave patterns (Figure 9c) agree very well with the measured $\mathrm{d} I / \mathrm{d} V$ spectral maps (Figure 9b) regarding both the lateral electron density distribution and the relative peak intensities. The remarkable agreement between theory and experiment is a clear indication that the physically relevant properties regarding confinement and scattering are well described by the simple model of rectangular areas featuring a constant effective potential for electron scattering. Consequently, creating a rich electronic structure by a supramolecularly engineered complex nanoarchitectures of quantum corrals provides an intrinsically parallel method to impose novel electronic properties on entire surfaces. Note that the controlled electron confinement can be used to steer the organization of single (magnetic) atoms ${ }^{[14]}$ and potentially similarly of molecular species, and that very recent results demonstrate tunable electron confinement via the open honeycomb metal-organic networks described above. ${ }^{[60]}$

\section{Conclusion}

In conclusion, a comprehensive overview of our recent progress towards systematic supramolecular design using dicarbonitrile-oligophenyl building blocks on well-defined, smooth metal surfaces has been presented. By employing purely organic layers, a flat adsorption geometry prevails, in marked contrast to unfunctionalised oligophenyls. The balance between epitaxial fit on the substrate atomic lattice and lateral interactions leads to a fascinating variety of open networks. Among them, a novel chiral kagomé topology is obtained, giving rise to a dichotomous electronic confinement. By cobalt-directed assembly of linear linkers we engineered highly regular open honeycomb lattices with an unusual threefold coordination of the metal centers using nitrile moieties. These networks are tunable with pore sizes spanning the $10-29 \mathrm{~nm}^{2}$ range and represent versatile templates for the organization of molecular guests or metal clusters, tunable electron confinement, and the control of supramolecular dynamers. Moreover, a reduction of the linker symmetry is sufficient to trigger a qualitative change from convergent to divergent assembly and entails the formation of disordered, glassy coordination networks. It is suggested that the insight gained with these systems contributes to a rationale for the bottom-up fabrication of surface-confined supramolecular nanostructures and layers with prospects for further novel physicochemical properties.

\section{Acknowledgements}

Work supported by the European Science Foundation Collaborative Research Program SONS2-FunSMARTs II, Center for Functional Nanostructures Karlsruhe (DFG-CFN Project E3.5), IGSSE and IAS at TU München, Munich Center for Advanced Photonics (MAP), ERC Advanced Grant MolArt ( $\left.{ }^{\circ} 247299\right)$. This article is part of a Special Issue on Multiscale Self-Organization of Functional Nanostructures.

Received: July 16, 2010

Revised: November 12, 2010

Published online: March 11, 2011

[1] a) J. M. Lehn, Proc. Natl. Acad. Sci. USA 2002, 99, 4763-4768P b) K. Ariga, J. P. Hill, M. V. Lee, A. Vinu, R. Charvet, S. Acharya, Sci. Technol. Adv. Mater. 2008, 9, 014109-014205; c) T. Kato, N. Mizoshita, K. Kishimoto, Angew. Chem. Int. Ed. 2006, 45, 38-68.

[2] a) T. Kato, Science 2002, 295, 2414-2418; b) J. W. Goodby, G. H. Mehl, I. M. Saez, R. P. Tuffin, G. Mackenzie, R. Auzély-Velty, T. Benvegnu, D. Plusquellec, Chem. Commun. 1998, 19, 2057-2070.

[3] a) J. V. Barth, G. Costantini, K. Kern, Nature 2005, 437, 671-679; b) N. Lin, S. Stepanow, F. Vidal, K. Kern, M. S. Alam, S. Strömsdörfer, V. Dremov, P. Müller, A. Landa, M. Ruben, Dalton Trans. 2006, 23, 2794-2800; c) J. A. A. W. Elemans, S. Lei, S. De Feyter, Angew. Chem. Int. Ed. 2009, 48, 7278-7332.

[4] a) O. Shekhah, H. Wang, M. Paradinas, C. Ocal, B. Schüpbach, A. Terfort, D. Zacher, R. A. Fischer, C. Wöll, Nat. Mater. 2009, 8, 481-484; b) G. Mercurio, E. R. McNellis, I. Martin, S. Hagen, F. Leyssner, S. Soubatch, J. Meyer, M. Wolf, P. Tegeder, F. S. Tautz, K. Reuter, Phys. Rev. Lett. 2010, 104, 036102-036106; c) W. Auwärter, K. Seufert, F. Klappenberger, J. Reichert, A. Weber-Bargioni, A. Verdini, D. Cvetko, M. Dell'Angela, L. Floreano, A. Cossaro, G. Bavdek, A. Morgante, A. P. Seitsonen, Johannes V. Barth, Phys. Rev. B 2010, 81, 245403; d) A. Schiffrin, A. Riemann, W. Auwärter, Y. Pennec, A. Weber-Bargioni, D. Cvetko, A. Cossaro, A. Morgante, J. V. Barth, Proc. Natl. Acad. Sci. USA 2007, 104, 5279-5284.

[5] J. A. Theobald, N. S. Oxtoby, M. A. Phillips, N. R. Champness, P. H. Beton, Nature 2003, 424, 1029-1031.

[6] M. E. Cañas-Ventura, W. Xiao, D. Wasserfallen, K. Müllen, H. Brune, J. V. Barth, R. Fasel, Angew. Chem. Int. Ed 2007, 46, 1814-1818.

[7] J. V. Barth, Annu. Rev. Phys. Chem 2007, 58, 375-407.

[8] F. Silly, M. R. Castell, Phys. Rev. Lett. 2005, 94, 046103.

[9] M. Ruben, Angew. Chem. Int. Ed. 2005, 44, 1594-1596.

[10] a) T. Yokoyama, S. Yokoyama, T. Kamikado, Y. Okuno, S. Mashiko, Nature 2001, 413, 619-621; b) J. V. Barth, J. Weckesser, C. Cai, P. Günter, L. Bürgi, O. Jeandupeux, K. Kern, Angew. Chem. Int. Ed. 2000, 39, 1230-1234.

[11] S. Stepanow, M. A. Lingenfelder, A. Dimitriev, H. Spillmann, E. Delvigne, N. Lin, X. Deng, C. Cai, J. V. Barth, K. Kern, Nat. Mater. 2004, 3, 229-233.

[12] A. Dmitriev, H. Spillmann, N. Lin, J. V. Barth, K. Kern, Angew. Chem. Int. Ed. 2003, 42, 2670-2673.

[13] a) N. Lin, S. Stepanow, M. Ruben, J. V. Barth, Top. Curr. Chem. 2009, 287, 1-44; b) S. Stepanow, N. Lin, J. V. Barth, J. Phys. Cond. Matt. 2008, 20, 184002.

[14] a) Y. Pennec, W. Auwärter, A. Schiffrin, A. Weber-Bargioni, A. Riemann, J. V. Barth, Nat. Nanotechnol. 2007, 2, 99-103; b) A. Schiffrin, J. Reichert, W. Auwärter, G. Jahnz, Y. Pennec, A. Weber-Bargioni, V. S. Stepanyuk, L. Niebergall, P. Bruno, J. V. Barth, Phys. Review B 2008, 78, 035424; c) V. S. Stepanyuk, N. N. Negulyaev, L. Niebergall, R. C. Longo, P. Bruno, Phys. Rev. Lett. 2006, 97, 186403. 
[15] S. Griessl, M. Lackinger, M. Edelwirth, M. Hietschold, W. M. Heckl, Single Mol. 2002, 3, 25-31.

[16] M. Ruben, D. Payer, A. Landa, A. Comisso, C. Gattinoni, N. Lin, J.-P. Collin, J.-P. Sauvage, A. De Vita, K. Kern, J. Am. Chem. Soc. 2006, 128, 15644-15651.

[17] G. Pawin, K. L. Wong, K. Y. Kwon, L. Bartels, Science 2006, 313, 961-962.

[18] S. L. Tait, A. Langner, N. Lin, R. Chandrasekar, O. Fuhr, M. Ruben, K. Kern, Chem. Phys. Chem. 2008, 9, 2495-2499.

[19] J. Stöhr, NEXAFS Spectroscopy and the Structure of Molecules Bonded to Surfaces, Springer, 1991.

[20] N. C. Greenham, S. C. Moratti, D. D. C. Bradley, R. H. Friend, A. B. Holmes, Nature 1993, 365, 628-630.

[21] M. Berggren, M. Granström, O. Inganäs, M. Andersson, Adv. Mater. 1995, 7, 900-903.

[22] W. Warta, N. Karl, Phys. Rev. B: Condens. Matter, 1985, 32, 1172.

[23] Handbook of Oligo- and Polythiophenes, (Ed: D. Fichou) Wiley-VCH, Weinheim, 1999

[24] S. R. Forrest, Chem. Rev. 1997, 97, 1793-1896.

[25] C. C. Leznoff, A. B. P. Lever, Phthalocyanines- Properties and Applications, Vol. 1-4, VCH, New York, USA 1989-1996.

[26] H. Klauk, Chem. Soc. Rev. 2010, 39, 2643-2666.

[27] a) M. Gsänger, J. H. Oh, M. Könemann, H. W. Höffken, A.-M. Krause, Z. Bao, F. Würthner, Angew. Chem. Int. Ed. 2010, 49, 643, cover picture; b) www.nikon.com, Nikon Horizons, 9, 03 (accessed May, 2009)

[28] Handbook of Conducting Polymers, 2nd ed. (Eds: T. Skotheim, R. L. Elsenbaumer, J. R. Reynolds) Marcel Dekker, New York 1998.

[29] N. O'Neill, Liq. Cryst. 2007, 34, 1307-1316.

[30] a) Y. Baudry, B. Baumeister, G. Das, D. Gerard, S. Matile, N. Sakai, A. Som, N. Sordé, P. Talukdar, Chimia 2002, 56, 667-671; b) H. Yin, G. Lee, K. A. Sedey, O. Kutzki, H. S. Park, B. P. Orner, J. T. Ernst, H.-G. Wang, S. M. Sebti, A. D. Hamilton, J. Am. Chem. Soc. 2005, 127, 10191-10196.

[31] a) S.-W. Hwang, Y. Chen, Macromolecules 2001, 34, 2981-2986; b) B. Liu, S. Wang, G. C. Bazan, A. Mikhailovsky, J. Am. Chem. Soc. 2003, 125, 13306-13307.

[32] a) I. Yamaguchi, K. Goto, M. Sato, Macromolecules 2009, 42, 78367845; b) I. Yamaguchi, K. Goto, M. Sato, Tetrahedron 2009, 65, $3645-3652$

[33] U. Schlickum, R. Decker, F. Klappenberger, G. Zoppellaro, S. Klyatskaya, W. Auwärter, K. Kern, H. Brune, M. Ruben, J. V. Barth, J. Am. Chem. Soc. 2008, 130, 11778-11782.

[34] D. Kühne, F. Klappenberger, R. Decker, U. Schlickum, H. Brune, S. Klyatskaya, M. Ruben, J. V. Barth, J. Phys. Chem. C 2009, 113, 17851-17859.

[35] a) K.-F. Braun, S.-W. Hla, Nano Lett. 2005, 5, 73-76; b) S. Müllegger, I. Salzmann, R. Resel, G. Hlawacek, C. Teichert, A. Winkler, J. Chem. Phys. 2004, 121, 2272.

[36] M. Marschall, J. Reichert, K. Seufert, W. Auwärter, F. Klappenberger, A. Weber-Bargioni, S. Klyatskaya, G. Zoppellaro, A. Nefedov, T. Strunskus, C. Wöll, M. Ruben, J. V. Barth, ChemPhysChem 2010, 11, 1446-1451.

[37] W. Chen, H. L. Zhang, H. Huang, L. Chen, A. T. S. Wee, Appl. Phys. Lett. 2008, 92, 193301-193303.

[38] F. Klappenberger, D. Kühne, M. Marschall, S. Neppl, W. Krenner, A. Nefedov, T. Strunskus, K. Fink, C. Wöll, S. Klyatskaya, O. Fuhr, M. Ruben, J. V. Barth, Adv. Funct. Mater. in press.

[39] R. Resel, J. Phys.: Condens. Matter. 2008, 20, 184009.
[40] a) B. J. Holiday, C. A. Mirkin, Angew. Chem. Int. Ed. 2002, 40, 2022-2043; b) O. M. Yaghi, M. O'Keeffe, N. W. Ockwig, H. K. Chae, M. Eddaoudi, J. Kim, Nature 2003, 423, 705-714; c) M. Ruben, J. Rojo, F. J. Romero-Salguero, L. H. Uppadine, J.-M. Lehn, Angew. Chem. Int. Ed. 2004, 43, 3644-3662.

[41] a) W. P. Fehlhammer, M. Fritz, Chem. Rev. 1993, 93, 1243-1280; b) N. A. Kato, M. Hara, H. Sasabe, W. Knoll, Nanotechnology 1996, 7, 122-127.

[42] S. Stepanow, N. Lin, D. Payer, U. Schlickum, F. Klappenberger, G. Zoppellaro, M. Ruben, H. Brune, J. V. Barth, K. Kern, Angew. Chem. Int. Ed. 2007, 46, 710-713.

[43] U. Schlickum, R. Decker, F. Klappenberger, G. Zoppellaro, S. Klyatskaya, M. Ruben, I. Silanes, A. Arnau, K. Kern, H. Brune, J. V. Barth, Nano Lett. 2007, 7, 3813-3817.

[44] D. Kühne, F. Klappenberger, R. Decker, U. Schlickum, H. Brune, S. Klyatskaya, M. Ruben, J. V. Barth, J. Am. Chem. Soc. 2009, 131, 3881-3883.

[45] U. Schlickum, F. Klappenberger, R. Decker, G. Zoppellaro, S. Klyatskaya, M. Ruben, K. Kern, H. Brune, J. V. Barth, J. Phys. Chem. C 2010, 114, 15602-15606.

[46] a) M. O. Blunt, J. C. Russell, M. del Carmen Giménez-López, J. P. Garrahan, X. Lin, M. Schröder, N. R. Champness, P. H. Beton, Science 2008, 322, 1077-1081; b) J. P. Garrahan, A. Stannard, M. O. Blunt, P. H. Beton, Proc. Natl. Acad. Sci. USA 2009, 106, 15209-15213.

[47] M. Marschall, J. Reichert, A. Weber-Bargioni, K. Seufert, W. Auwärter, S. Klyatskaya, G. Zoppellaro, M. Ruben, J. V. Barth, Nat. Chem., 2010, 2, 131-137.

[48] Z. Nie, E. Kumacheva, Nat. Mater. 2008, 7, 277-278.

[49] T. Kudernac, S. Lei, J. A. A. W. Elemas, S. De Feyter, Chem. Soc. Rev. 2009, 38, 402-421.

[50] X. Ma, H. L. Meyerheim, J. Barthel, J. Kirchner, S. Schmitt, E. Umbach, Appl. Phys. Lett. 2004, 84, 4038-4040.

[51] J. V. Barth, Surf. Sci. 2009, 603, 1533-1541.

[52] P.Gambardella, S.Stepanow, A. Dmitriev, J. Honolka, F. M. F. deGroot, M. Lingenfelder, S. S. Gupta, D. D. Sarma, P. Bencok, S. Stanescu, S. Clair, S. Pons, N. Lin, A. P. Seitsonen, H. Brune, J. V. Barth, K. Kern, Nat. Mater. 2009, 8, 189-193.

[53] R. Decker, U. Schlickum, F. Klappenberger, G. Zoppellaro, S. Klyatskaya, M. Ruben, J. V. Barth, H. Brune, Appl. Phys. Lett. 2008, 93, 243102-3.

[54] P. Gambardella, S. Rusponi, M. Veronese, S. S. Dhesi, C. Grazioli, A. Dallmeyer, I. Cabria, R. Zeller, P. H. Dederichs, K. Kern, C. Carbone, H. Brune, Science 2003, 300, 1130-1133.

[55] U. Heiz, W. D. Schneider, Crit. Rev. Solid State Mater. Sci. 2001, 26, 251-290

[56] V. Balzani, A. Credi, M. Venturi, Chem. Phys. Chem. 2008, 9, 202-220.

[57] D. Kühne, F. Klappenberger, W. Krenner, S. Klyatskaya, M. Ruben, J. V. Barth, Proc. Natl. Acad. Sci. USA 2010, 107, 21332-21336.

[58] J. Li, W.-D. Schneider, S. Crampin, R. Berndt, Surf. Sci. 1999, 422, 95-106.

[59] a) F. Klappenberger, D. Kühne, W. Krenner, I. Silanes, A. Arnau, F.J. García de Adajo, S. Klyatskaya, M. Ruben, J. V. Barth, Nano Lett. 2009, 9, 3509-3514; b) J. Lobo-Checa, M. Matena, K. Müller, J. H. Dil, F. Meier, L. H. Gade, T. Jung, M. Stöhr, Science 2009, 325, 300-303.

[60] F. Klappenberger, D. Kühne, W. Krenner, I. Silanes, A. Arnau, F. J. García de Abajo, S. Klyatskaya, M. Ruben, J. V. Barth, Phys. Rev. Lett. 2011, 106, 026802. 